\title{
EVALUATION OF CLUSTERED MICROCALCIFICATIONS, INITIALLY GRADED AS LIKELY MALIGNANT ON BASELINE MAMMOGRAPHY, WITH STEREOTACTIC BIOPSY: A RAD-PATH CORRELATION
}

\author{
Hafsa S. Babar ${ }^{1}$, Anis-ur-Rehman ${ }^{1}$, Ali Zargham Babar ${ }^{2}$, Imran Khalid Niazi ${ }^{1}$ \\ ${ }^{1}$ Department of Radiology, Shaukat Khanum Memorial Cancer Hospital and Research Centre, Lahore, ${ }^{2}$ King Edward \\ Medical University, Lahore, Pakistan
}

Received: 30 January 2019 / Accepted: 18 March 2019

\begin{abstract}
Background: Stereotactic-guided breast biopsy is an invincible tool to sample abnormalities visible only on mammography with subtle or occult ultrasound findings. Common mammographic abnormalities which require stereotactic core biopsy include calcifications, architectural distortion and satellite lesions. With advancement in techniques for adequate localisation of lesions, the use of stereotactic needle breast biopsy is exponentially increasing. A study was performed to look for frequency of benignancy or malignancy of microcalcifications and architectural distortions in patients initially falling in malignant category of Breast Imaging-Reporting and Data System of American College of Radiology (BI-RADS ${ }^{\circledR}$ ) Assessment Categories.
\end{abstract}

Materials and Methods: Patient presenting in outpatient (OPD) clinic who underwent stereotactic biopsies for labelled malignant on mammograms from May 2015 to May 2018 was included in retrospective study. Age, technique used, baseline mammogram and histopathology were reviewed.

Results: A total of 91 patients underwent stereotactic biopsy. Age range varied between 28 and 81 years. Stereotacticguided biopsies were taken using a standard 14-gauge core needle with long throw of $22 \mathrm{~mm}$ excursion. None of the biopsy was inconclusive. In comparison with baseline mammograms and histopathology, $40 \%$ of the clusters of microcalcification which were initially labelled as malignant turned out to be malignant on histopathology as well, whereas $60 \%$ were reported as benign breast parenchyma.

Conclusion: Agreement between the diagnostic accuracy of microcalcifications on mammogram and histopathology of same lesions after stereotactic biopsy was comparable to international figures with probability of being benign surpassing malignant on routine screening.

Key words: Breast cancer, mammography, microcalcifications, stereotactic biopsy

\section{Introduction}

With recent advancement in imaging techniques and increased levels of patient awareness and education, a large population of women, most of them 40 years or older, undergo screening mammography. This has led to increased detection of the early and often non-palpable breast cancer, reducing overall breast cancer mortality and

Correspondence: Dr. Hafsa S. Babar, Department of Radiology, Shaukat Khanum Memorial Cancer Hospital and Research Centre,

Lahore, Pakistan. Email: hafsababar_90@hotmail.com morbidity over the past few decades..$^{[1,2]}$

The field of breast interventions has profited immensely from recent evolution in imaging. Pointing out the lesion, however, is only the starting point. Once the lesion is perceived, the challenge is to then aptly diagnose it in as minimally invasive a way as possible. This, in turn, determines the course of patient's entire management.

In the recent past, tomosynthesis, a latest 3-D mammographic technique, has proved itself in increasing the diagnostic 
accuracy of breast cancer detection rates. It is predicted that tomosynthesis will lead digital mammography in the upcoming years. In patients with suspicious breast lesions identified on baseline mammography or tomosynthesis, stereotactic-guided biopsy is one of the many available options for obtaining the tissue essential for histopathological diagnosis. Usually, the lesions undergoing stereotactic biopsies are occult on ultrasound and typically present with microcalcification or an area of architectural distortion detected on mammography.

Clustered suspicious microcalcifications can be a very early sign of malignancy, particularly in ductal carcinoma in situ (DCIS). Microcalcifications are usually detected on screening mammographies, thus require stereotactic guidance for biopsy. ${ }^{[2,3]}$

In this paper, we aim to present our biopsy method and retrospectively evaluate the results and compare them with the final histopathological diagnosis in all patients undergoing stereotactic-guided biopsies performed over a 3-year period in our tertiary care oncological setup.

\section{Materials and Methods}

Between May 2015 and May 2018, 91 patients were referred to our clinic for stereotactic biopsy. These patients had non-palpable microcalcifications, sonographically occult architectural distortions or mass lesions for which they underwent a stereotactic biopsy. Biopsy was successfully performed in all 91 of these patients. Of these, 10 patients were excluded because biopsy was performed for mass/architectural distortions and not microcalcifications. The remaining 81 patients (median age 53 years, range $28-81$ years) were included in the study. Biopsy was performed for 85 lesions, all of which were mammographically detected and could not be identified on US. Informed consent forms regarding the procedure of biopsy and academic use of data were signed by all patients. The institutional review board did not ask for any further approval as this is a retrospective analysis of the results [Figures 1 and 2].

Before biopsy, lesions were classified according to the new edition of mammographic BI-RADS classification published in 2015 [Table 1]. 21 lesions comprising amorphous calcifications were labelled as BI-RADS

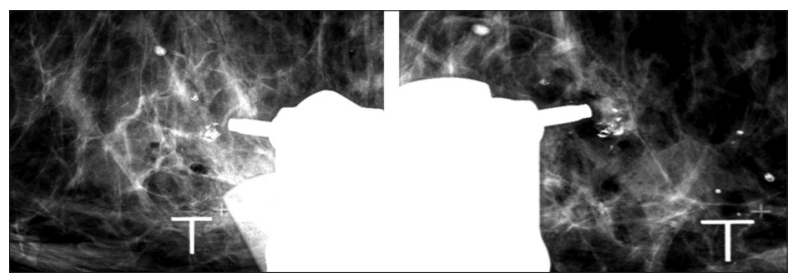

Figure 1: Images from stereotactic biopsy performed with targeting callipers placed on the lesion. Pre-fire image pair at $-15^{\circ}$ and $+15^{\circ}$ from midline

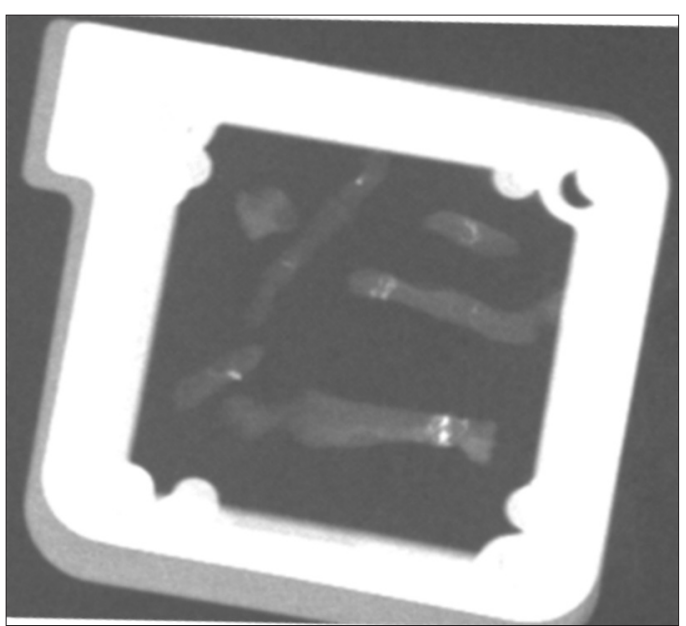

Figure 2: Post-specimen radiograph demonstrates microcalcification indicating adequate sampling

4A and 25 lesions comprising coarse heterogeneous calcifications were characterised as BI-RADS category 4B (87.38\% in total). 35 lesions comprising fine pleomorphic, fine linear or branching calcifications were classified as BI-RADS category $4 \mathrm{C} / 5$.

We had no standard prebiopsy restrictions, patients with deranged coagulation profile and those on anticoagulants were screened. Some centres prefer to stop administering blood-thinning agents before biopsy. Patients are instructed to discontinue aspirin and NSAIDs 1 week before the procedure. An international normalised ratio level of $<1.5$ is confirmed on the day of the biopsy.

Lesions that pose difficulty to biopsy under stereotactic guidance include lesions that are lying very deep in the breast, are high in the upper outer quadrant and overall smaller breast size.

Biopsies were performed using a prone biopsy table (Multicare Platinum; Hologic) with 14-gauge (BARD) 
biopsy device having $22 \mathrm{~mm}$ excursion. Five cores were taken in each case. The best approach to the lesion was established by reviewing the mammogram beforehand. Craniocaudal and mediolateral views were taken before targeting the lesion. After acquiring the scout view, paired $(+15$ and -15$)$ images were obtained for accurate needle placement using the $x-y-z$ coordinates determined by the machine. Then, a local anaesthetic was applied and the needle was parked in the breast. Further, pre- and post-fire stereo images were acquired. The whole procedure took around $30 \mathrm{~min}$ which decreased further as our experience increased and we modified our technique over the time. Most of the patients tolerated the procedure really well. Post-biopsy tissue specimen was placed in a Petri dish, and radiography was performed. Specimen graphy was acquired in all lesions. The biopsy of a microcalcification cluster was rendered technically adequate if microcalcifications were identified histologically and/or on the radiograph of the specimen. There were no specific post-biopsy care instructions.

After a discussion at the multidisciplinary team meeting, patients with a histologic diagnosis of atypia, infiltrating or in situ carcinoma and surgical excision were recommended. Mammographic follow-up was recommended for the remaining patients with benign biopsy results. All patients had at least 1 year of follow-up.

\section{Results}

Stereotactic-guided biopsy results revealed 32 (39.8\%) malignant lesions including DCIS (22.2\%), infiltrative ductal carcinoma (14.8\%) and infiltrative lobular carcinoma $(0.02 \%)$. Findings at ensuing surgery confirmed malignancy in all 32 lesions. 3 (4\%) of the lesions were

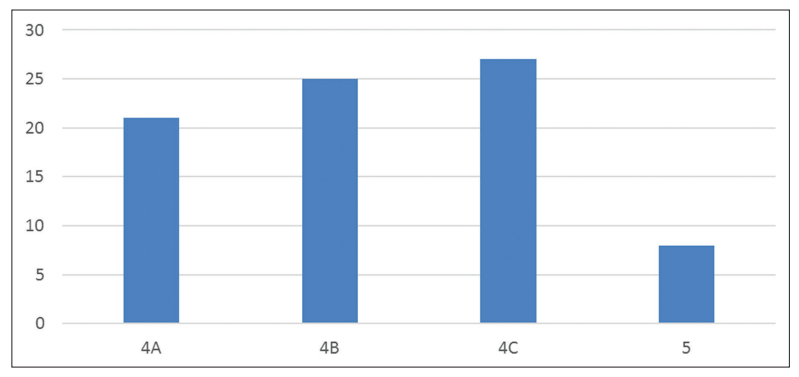

Table 1: Bar chart displays the various categories that were assigned to all the lesions that ultimately were subjected to stereotactic biopsy atypical ductal hyperplasia and none were atypical lobular hyperplasia. No atypical hyperplasia lesions were upgraded to invasive carcinoma. The histologic results were benign in $46(60.2 \%)$ of 81 lesions. None of the biopsies were inconclusive. Repeat biopsies were performed in four cases where the suspicion of malignancy was high. Out of the benign lesions, stromal hyperplasia dominated and accounted for 16 lesions (34.7\%), fibrosisadenosis made up 13 (28.2\%) of the lesions. 05 (10.8\%) of these were sclerosis adenosis and fibrocystic changes each. Fat necrosis was found in $4(8.69 \%)$ lesions, whereas lactational changes were diagnosed in $1(2.17 \%)$ case. It was no surprise to find fibroadenomatoid lesions in $2(4.34 \%)$ of the clustered microcalcifications [Table 2].

\section{Discussion}

The main aim of performing a percutaneous biopsy is to be able to formulate an apt histologic diagnosis by obtaining an emblematic sample from what may be a radiologically suspicious lesion. We want to circumvent a false-negative biopsy result and also to minimise the chance of underestimation in case of DCIS and atypia.

Image-guided percutaneous breast biopsy is a costeffective and safe method of formulating an accurate

\section{Table 2: Histologic diagnoses on stereotactic breast biopsy}

\begin{tabular}{l|c}
\hline Histologic diagnoses & $\boldsymbol{n}(\%)$ \\
\hline Benign lesions $(n=46)$ & \\
\hline Fibrosis-adenosis & $13(16)$ \\
\hline Fibroadenoma and fibroadenomatoid lesions & $2(2.5)$ \\
\hline Fibrocystic changes & $5(6)$ \\
\hline Sclerosing adenosis & $5(6)$ \\
\hline Benign stromal hyperplasia & $16(20)$ \\
\hline Fat necrosis & $4(5)$ \\
\hline Lactational changes & $1(1)$ \\
\hline Malignant lesions $(n=32)$ & \\
\hline DCIS & $18(22)$ \\
\hline IDC & $12(15)$ \\
\hline ILC & $2(2.5)$ \\
\hline Atypical lesions $(n=3)$ & \\
\hline Atypical ductal hyperplasia & $3(4)$ \\
\hline
\end{tabular}

DCIS: Ductal carcinoma in situ, ILC: Invasive lobular carcinoma, IDC: Invasive ductal carcinoma 
tissue diagnosis of breast abnormalities. It has replaced surgical biopsy as the modus operandi for establishing diagnosis of most breast lesions, precluding surgery when benign pathology results are demonstrated or allowing for definitive upfront surgical treatment in most malignant cases.

Approximately $70-90 \%$ of the 1.6 million breast biopsies performed in 1 year, in the US are imageguided percutaneous procedures. Stereotactic, US and MR guidance are all reassuring methods for performing image-guided biopsy. US-guided biopsy is the preferred method due to its speed, cost, patient comfort and real-time capabilities. However, for sonographically occult lesions, microcalcifications and architectural distortions which are not well-visualised sonographically, stereotactic biopsy is the favoured method. With all biopsy methods, careful attention to technique and radiologic-pathologic concordance are needed for a positive outcome. ${ }^{[1,2]}$

Stereotactic-guided biopsy is an outpatient procedure where general anaesthesia is not necessary and is less invasive, carrying almost no risks. Considering that significant number of women is undergoing screening mammography, an increased number of breast biopsies are being recommended. It is usually expected that most of these lesions are benign and this minimally invasive technique spares patients from undergoing expensive and potentially deforming surgeries.

In our study, we basically categorised various microcalcifications in both cancer and non-cancer patients, according to the latest mammographic BI-RADS classification published in 2015. These patients ultimately underwent stereotactic biopsies, and then, we correlated their histological diagnosis to see the extent to which these lesions were adequately classified radiologically.

Various factors that were considered during our trial included the number of specimens, radiograph after the procedure to assess if correct set of calcifications has been sampled. Obtaining large numbers of specimens were not that useful for an accurate diagnosis, it was an adequate number of correctly targeted specimens which is more beneficial. ${ }^{[4]}$
Radiography of the specimen for the detection of microcalcifications was introduced by Meyer et al. ${ }^{[5]}$ and Lieberman et $a{ }^{[6]}$ and is an indicator of adequate sampling of the targeted microcalcifications. At times, microcalcifications were not seen on the post-procedure radiography, however, were diagnosed histopathologically.

The addition of digital add-on mammography and the vacuum-assisted needles to stereotactic breast biopsy equipment have immensely helped in decreasing procedure duration and the tissue sampling errors. It also offers the advantage of lower $\operatorname{cost}^{[7]}$ and requires less space than a dedicated prone unit. Moreover, the setup can be utilised to do routine mammography when biopsy procedures are not being done.

In addition to this, with the introduction and increased clinical use of digital breast tomosynthesis, there is improved visualisation of masses and architectural distortions. There is substantial evidence that dialectical behaviour therapy (DBT) leads to a higher breast cancer detection rate by $31 \%{ }^{[8]}$ over that with conventional full-field digital mammography. DBT along with the vacuum-assisted needle is effective in retrieving microcalcifications, leading to adequate sampling and increased cancer detection overall. ${ }^{[2]}$

Our results indicate that stereotactic-guided breast biopsies are safe and cost-effective method of establishing a diagnosis and can be performed effectively without any substantial risks. Most of the microcalcifications identified on digital mammography fall in benign category even in cancer patients. However, the mammograms labelled as highly suspicious or those classified as category $4 \mathrm{C} / 5$ almost always turned out to be malignant.

We also concluded that proper visualisation of the microcalcifications on the preprocedural radiographs of the breast and adequate positioning of the biopsy needle before obtaining tissue samples adds more substance to successfully retrieve microcalcifications, than is extraction of a larger number of tissue samples.

\section{Conflict of Interest}

The authors declare that they have no conflict of interest. 


\section{References}

1. Mahoney MC, Newell MS. Breast intervention: How I do it. Radiology 2013;268:12-24.

2. Schrading S, Distelmaier M, Dirrichs T, et al. Digital breast tomosynthesis-guided vacuum-assisted breast biopsy: Initial experiences and comparison with prone stereotactic vacuum-assisted biopsy. Radiology 2015;274:654-62.

3. Jackman RJ, Burbank F, Parker SH, et al. Stereotactic breast biopsy of nonpalpable lesions: Determinants of ductal carcinoma in situ underestimation rates. Radiology 2001; 218:497-502.

4. Jackman RJ, Rodriguez-Soto J. Breast microcalcifications: Retrieval failure at prone stereotactic core and vacuum breast biopsy - frequency, causes, and outcome. Radiology 2006; 239:61-70.

4. Parker SH, Lovin JD, Jobe WE, et al. Stereotactic breast biopsy with a biopsy gun. Radiology 1990;176:741-7.

5. Meyer JE, Lester SC, Frenna TH, et al. Occult breast calcifications sampled with large-core biopsy: Confirmation with radiography of the specimen. Radiology 1993;188:581-2.

6. Liberman L, Evans WP $3^{\text {rd }}$, Dershaw DD, et al. Radiography of microcalcifications in stereotaxic mammary core biopsy specimens. Radiology 1994;190:223-5.

7. Welle GJ, Clark M, Loos S, et al. Stereotatic breast biopsy. Am J Roentgenol 2000;175:59-63.

8. Freer PE, Niell B, Rafferty EA. Preoperative tomosynthesisguided needle localization of mammographically and sonographically occult breast lesions. Radiology 2015;275: $377-83$.

\section{Authorship Contributions}

Concept and Design: HSB, AR, AZB, IKN; Data Collection and interpretation: HSB, AZB; Literature review and writing: HSB, AR, IKN; Manuscript approval: HSB, AR, AZB, IKN 